\title{
SYMPLECTIC HOMOGENEOUS SPACES
}

BY

\author{
SHLOMO STERNBERG(1)
}

\begin{abstract}
In this paper we make various remarks, mostly of a computational nature, concerning a symplectic manifold $X$ on which a Lie group $G$ acts as a transitive group of symplectic automorphisms. The study of such manifolds was initiated by Kostant [4] and Souriau [5] and was recently developed from a more general point of view by Chu [2].

The first part of this paper is devoted to reviewing the Kostant, Souriau, Chu results and deriving from them a generalization of the Cartan conjugacy theorem. In the second part of this paper we apply these results to Lie algebras admitting a generalized $(k, p)$ decomposition.
\end{abstract}

In this paper we make various remarks, mostly of a computational nature, concerning a symplectic manifold $X$ on which a Lie group $G$ acts as a transitive group of symplectic automorphisms. The study of such manifolds was initiated by Kostant [4] and Souriau [5] and was recently developed from a more general point of view by Chu [2]. For the convenience of the reader we will begin by summarizing the basic facts.

1. General facts. Let $G$ be a Lie group and $X=G / H$ a homogeneous space for $G$ where $H$ is a closed subgroup, and let $\pi: G \rightarrow G / H=X$ be the projection. If $\Omega$ is an invariant form on $X$ then it is clear that $\sigma=\pi^{*} \Omega$ is a left invariant form on $G$ which satisfies

(i) $\xi\lrcorner \sigma=0$ for all $\xi \in h$ where $h$ is the Lie algebra of $H$;

(ii) $\sigma$ is invariant under right multiplication by elements of $H$, and hence under Ad for elements of $H$.

Conversely, it is clear that any left invariant form $\sigma$ on $G$ satisfying (i) and (ii) arises from $G / H$. If $\Omega$ is a symplectic form then it is clear that a left invariant vector field will satisfy $\xi\lrcorner \sigma=0$ if and only if $\xi \in h$. Furthermore, since $d \sigma=0$, the set of all vector fields satisfying $\xi\lrcorner \sigma=0$ forms an integrable subbundle of $T G$, and in particular, the left invariant ones form a subalgebra of the Lie algebra of $G$; let us call it $h_{\sigma}$. We have thus recovered $h$. Let $H_{\sigma}$ be the group generated by $h_{\sigma}$. Notice that for any $\xi \in h_{\sigma}$ we have $\left.\left.D_{\xi} \sigma=\xi\right\lrcorner d \sigma+d(\xi\lrcorner \sigma\right)=0$ so that $\sigma$ is invariant under $H_{\sigma}$. The only problem is that $H_{\sigma}$ need not be closed. Let

Received by the editors May 23, 1974.

AMS (MOS) subject classifications (1970). Primary 53C15, 53C30.

(1)This research was partially supported by NSF Grant GP43613X. 
us say that $\sigma$ is regular if $H_{\sigma}$ is closed. Notice that if $G / H$ is a symplectic homogeneous space, so that $H$ is a closed subgroup, and if we construct $\sigma$ as above, then $H_{\sigma}$ is just the connected component of the identity in $H$ (and hence a closed subgroup of $G$ ). We have thus established

Proposition 1.1 ( $\mathrm{CHU}$ [2]). Each 2p-dimensional homogeneous space determines a left invariant regular closed two form of rank $2 p$ on $G$. Conversely a regular left invariant closed two form determines a homogeneous symplectic space.

Notice that if $\sigma=d \beta$ where $\beta$ is a left invariant one form then $\sigma$ is automatically regular. Indeed $\left.\left.\left.D_{\xi} \beta=\xi\right\lrcorner d \beta+d(\xi\lrcorner \beta\right)=\xi\right\lrcorner \sigma$ for any left invariant vector field $\xi$ since $\xi\lrcorner \beta$ is constant. Thus $\xi \in h_{\sigma}$ if and only if $D_{\xi} \beta=0$. Let $\mathrm{Ad}^{\#}$ denote the coadjoint representation of $G$ on $g^{*}$. Now $H=\left\{a \mid\left(\operatorname{Ad}^{\#} a\right) \beta=\right.$ $\beta$ \} is clearly a closed subgroup and $H_{\sigma}$ is the identity component. In any event, it is clear from the foregoing discussion that

Proposition 1.2 (Kirillov-Kostant-Souriau). Each orbit, $\operatorname{Ad}^{\#}(G) \beta$ for $\beta \in g^{*}$ is a symplectic manifold whose symplectic structure is induced from $d \beta$.

We now assert

Proposition 1.3 (CHU [2]). If $G$ is a simply connected Lie group then every left invariant closed two form is regular.

We sketch the proof. Let $\sigma$ be a left invariant closed two form. We think of $\sigma$ as a one cocycle $f$ from $g$ to $g^{*}$, where $f(\xi)=\xi \downarrow \sigma$. Here $f$ is a cocycle relative to the action ad ad $^{\#}$ on $g^{*}$. Hence $f$ defines an action of $g$ as affine transformations on $g^{*}$ via

$$
\left.\xi \cdot \theta=\left(\operatorname{ad}^{\#} \xi\right) \theta+f(\xi)=\left(\operatorname{ad}^{\#} \xi\right) \theta+\xi\right\lrcorner \sigma .
$$

Since $G$ is simply connected this defines an affine action of $G$ on $g^{*}$. It is clear that $\xi \in h_{\sigma}$ if and only if $\xi \cdot \theta=0$. Thus $H_{\sigma}$ is the identity component of the isotropy group of the origin and hence closed.

Let $\sigma$ be a left invariant closed two form on $G$ and suppose that the subalgebra $h$ has minimum dimension among all subalgebras of the form $h_{\sigma}$. This implies that if $\sigma_{t}$ is a curve of closed one forms with $\sigma_{0}=\sigma$, then any $\xi \in h_{\sigma}$ can be extended to a curve $\xi_{t}$ with $\xi_{0}=\xi_{0}$ and $\xi_{t} \in h_{\sigma_{t}}$. (Indeed, choose a subspace $m$ complementary to $h_{\sigma}$ in the Lie algebra $g_{e}$. Since $\operatorname{dim} h_{\sigma}$ is minimal, this implies that $\operatorname{dim} h_{\sigma}=\operatorname{dim} h_{\sigma^{\prime}}$ for all $\sigma^{\prime}$ close to $\sigma$. Then projection along $m$ defines an isomorphism of $h_{\sigma}$ with $h_{\sigma^{\prime}}$ for all $\sigma^{\prime}$ close to $\sigma$.) In particular, 
let $\theta$ be any left invariant one form and consider $\sigma_{t}=\sigma+t d \theta$. We can write $\xi_{t}=\xi+t \xi^{\prime}+O\left(t^{2}\right)$. Examining the coefficient of $t$ in the equation $\xi_{t} \sqcup \sigma_{t}=0$ gives $\left.\xi\lrcorner d \theta+\xi^{\prime}\right\lrcorner \sigma=0$. Let $\eta$ be some other element of $h_{\sigma}$ and take the interior product of this last equation with $\eta$. The term $\left.\left.\left.\eta\lrcorner \xi^{\prime}\right\lrcorner \sigma=\xi^{\prime}\right\lrcorner \eta\right\lrcorner \sigma=0$ and we get $\eta\lrcorner \xi\lrcorner d \theta=0$. Now, since $\eta$ and $\theta$ are both left invariant, $\eta\lrcorner \theta$ is a constant and therefore

$$
\left.\left.\left.\left.\left.\left.0=D_{\xi}(\eta\lrcorner \theta\right)=D_{\xi} \eta\right\lrcorner \theta+\eta\right\lrcorner D_{\xi} \theta=[\xi, \eta]\right\lrcorner \theta+\eta\right\lrcorner \xi\right\lrcorner d \theta,
$$

since $\xi\lrcorner \theta$ is also constant. Thus $[\xi, \eta] \theta=0$. Since this holds for arbitrary $\theta$ we conclude that $[\xi, \eta]=0$. We have thus proved

Proposition 1.4. Let $\sigma$ be a left invariant closed two form such that $h_{\sigma}$ has minimal dimension. Then $h_{\sigma}$ is commutative. In particular, let $X$ be a homogeneous symplectic manifold of $G$ with maximal dimension. Then the connected component of the isotropy group of any point of $X$ is commutative.

For the case that $\sigma=d \theta$ is an exact two form this result was obtained by Duflo and Vergne [3]. (It is just a trivial observation to remark that their proof works just as well for the case of closed two forms.) For the case where $G$ is a semisimple group, the dual of the Lie algebra can be identified with the Lie algebra via the Killing form. In this case, to say that $h_{d \theta}$ has minimal dimension becomes the assertion that the centralizer of the corresponding element $\theta$ has minimal dimension, and Proposition 1.4 reduces to the classical assertion that for such regular elements the centralizer is abelian. For regular elements the subalgebra $h_{d \theta}$ is a Cartan subalgebra.

For semisimple subalgebras one has a conjugacy theorem for Cartan subalgebras which, in the real case, can be formulated as asserting that if $\theta$ is generic, then $h_{d \theta^{\prime}}$ is conjugate to $h_{d \theta}$ under the adjoint group if $\theta^{\prime}$ is sufficiently close to $\theta$. One can ask to what extent this remains true in the general case. This problem was pointed out to me by Michèle Vergne. It is not true for all Lie algebras as is shown by the following example: let $g=\mathbf{R}+V$ where $V$ is the trivial Lie algebra (a vector space with trivial bracket) and $[r, v]=r$ for $r \in \mathbf{R}$ and $v \in V$. It is easy to see that for any $\theta \in g^{*}$ which does not vanish on $V$ the subalgebra $h_{d \theta}$ consists of the hyperplane in $V$ defined by the equation $\theta(v)=0$, corresponding to two dimensional orbits in $g^{*}$. It is clear that no two such subalgebras are conjugate to one another if they are distinct. Let us call a $\theta$ in $g^{*}$ stable if $h_{d \theta^{\prime}}$ is conjugate to $h_{d \theta}$ for all $\theta^{\prime}$ close to $\theta$.

PROPOSITION 1.5. Suppose that $h_{d \theta}$ has minimal dimension and that $\left[g, h_{d \theta}\right] \cap h_{d \theta}=\{0\}$. Then $\theta$ is stable and conversely. 
Proof. It is clear that for any $\theta^{\prime}$ on the orbit of $\theta$ the algebra $h_{d \theta}$ and $h_{d \theta^{\prime}}$ are conjugate. Thus we will be done if we can find a submanifold $W$ transversal to the orbit through $\theta$ with the property that $h_{d \theta^{\prime}}=h_{d \theta}$ for all $\theta^{\prime} \in W$ (near $\theta$ ). By the implicit function theorem we can reduce the problem to the corresponding infinitesimal problem: to show that every $\theta^{\prime}$ can be written as $\theta_{1}+\theta_{2}$ where $\left.\theta_{1} \in g\right\lrcorner d \theta$ (the tangent space to the orbit) and $\left.h_{d \theta}\right\lrcorner d \theta_{2}=0$ (which, on account of the minimality of $\operatorname{dim} h_{d \theta}$, is the same as saying that $h_{d\left(\theta+\theta_{2}\right)}=h_{d \theta}$ if $\theta_{2}$ is sufficiently small). It therefore suffices to show that no vector in $g$ can be annihilated by all such $\theta_{1}$ and $\theta_{2}$. Now to say that $\langle\xi, g\lrcorner d \theta\rangle=0$ is the same as saying that $\xi\lrcorner d \theta=0$, i.e. that $\xi \in h_{d \theta}$. To say that $\left\langle\xi, \theta_{2}\right\rangle=0$ for all $\theta_{2}$ means that $\left\langle\xi, \theta_{2}\right\rangle=0$ for all $\theta_{2}$ with the property that $\left\langle\left[g, h_{d \theta}\right], \theta_{2}\right\rangle=0$, i.e. that $\xi \in\left[g, h_{d \theta}\right]$. By hypothesis this implies that $\xi=0$.

If $\theta$ is stable, then $h_{d \theta}$ must have the generic dimension, which is the minimal dimension. Suppose that there is some $\eta$ in $h_{d \theta}$ with $0 \neq[\eta, \zeta]$ in $h_{d \theta}$ for some $\zeta$ in $g$. Choose $\gamma$ with $\langle[\eta, \zeta], \gamma\rangle \neq 0$. If we apply the condition for the existence of a conjugacy of $h_{d(\theta+t \gamma)}$ with $h_{d \theta}$ and compare coefficients of $t$, it is easy to see that we must be able to solve the equations

$$
\langle[\xi, \eta], \zeta, \theta\rangle=\langle[\eta, \zeta], \gamma\rangle
$$

for all $\zeta$ in $g$. Choosing $[\eta, \zeta] \in h_{d \theta}$ and using Jacobi's identity on the left gives zero while the right side does not vanish, giving a contradiction.

Observe that Proposition 1.5 is not true if we replace the coboundary $d \theta$ by a cocycle $\sigma$. Indeed, consider the trivial three dimensional algebra. Here every two form is a cocycle and, for nonzero $\sigma$, the subalgebra $h_{\sigma}$ consists of the line $\xi\lrcorner \sigma=0$, and no distinct lines are conjugate since the adjoint group acts trivially. On the other hand, $[g, g]=0$, so the condition $\left[g, h_{\sigma}\right] \cap h_{\sigma}=0$ is certainly satisfied.

In order to understand this example it is useful to observe that for any Lie algebra $g$, we can form the central extension of $g$ by $H^{2}(g)$ as follows: choose a basis $c_{1}, \ldots, c_{k}$ for $H^{2}(g)$ and cycles $z_{1}, \ldots, z_{k}$ representing the $c$ 's. Then define

$$
[(v, x),(w, y)]=\left(z_{1}(x, y) c_{1}+\ldots+z_{k}(x, y) c_{k},[x, y]\right)
$$

where $v$ and $w$ are in $H^{2}(g)$ and $x$ and $y$ are in $g$. This gives a Lie algebra structure to $H^{2}(g)+g$.

If $\theta \in\left(H^{2}(g)+g\right)^{*}$ is given by $\theta((v, x))=a_{i}$ where $v=\Sigma a_{i} c_{i}$, then it is clear that $d \theta=z_{i}$. In this way every cocycle of $g$ can be regarded as a coboundary in the extended algebra. If $\sigma$ is a cocycle of $g$ corresponding to the coboundary $d \theta$ of the extended algebra, it is clear that $h_{d \theta}=H^{2}(g)+h_{\sigma}$. If $\sigma$ is stable 
then so is $\theta$ and conversely. We must therefore require the stability criterion in the extended algebra.

2. Computation of cocycles for algebras with decomposition. We would now like to classify the homogeneous symplectic manifolds for various interesting Lie groups. We will do this by reducing the problem to studying the behavior of closed two forms with respect to certain subgroups. In particular, we will make the following assumption about the Lie algebra $g$ of $G$. We will assume that there are two subspaces, $k$ and $p$, of $g$ such that

$$
g=k+p, \quad k \cap p=\{0\}, \quad[k, k] \subset k, \text { and }[k, p] \subset p .
$$

Thus we are assuming that $k$ is a subalgebra of $g$ and that $p$ is a supplementary subspace to $k$ which is stable under the action of $k$. We do not make any special assumptions at the moment about $p$. Thus $[p, p]$ will have both a $k$ and a $p$ component which we denote by $r$ and $s$ respectively: for $\eta$ and $\eta^{\prime}$ in $p$ we have $\left[\eta, \eta^{\prime}\right]=r\left(\eta, \eta^{\prime}\right)+s\left(\eta, \eta^{\prime}\right)$ where $r\left(\eta, \eta^{\prime}\right) \in k$ and $s\left(\eta, \eta^{\prime}\right) \in p$. Jacobi's identity implies some identities on $r$ and $s$. It is easy to check that these are

$$
\notin r\left(s\left(\eta, \eta^{\prime}\right), \eta^{\prime \prime}\right)=0, \quad \&\left\{s\left(s\left(\eta, \eta^{\prime}\right), \eta^{\prime \prime}\right)+\left[r\left(\eta, \eta^{\prime}\right), \eta^{\prime \prime}\right]\right\}=0
$$

where $\&$ denotes cyclic sum. Also

$$
\left[\xi, r\left(\eta, \eta^{\prime}\right)\right]=r\left(\xi \cdot \eta, \eta^{\prime}\right)+r\left(\eta, \xi \cdot \eta^{\prime}\right)
$$

where $\xi \in k$ and $\eta, \eta^{\prime} \in p$ and we have written $\xi \cdot \eta$ for $[\xi, \eta]$, thinking of $k$ acting on $p$. We also have the equation

$$
\xi \cdot s\left(\eta, \eta^{\prime}\right)=s\left(\xi \eta, \eta^{\prime}\right)+s\left(\eta, \xi \cdot \eta^{\prime}\right)
$$

In addition we have the identity asserting that $k$ acts as a Lie algebra of linear transformations on $p$ and Jacobi's identity for $k$. Conversely, starting from any action of a Lie algebra $k$ on a vector space $p$ together with $r$ and $s$ satisfying the above identities it is clear that $g=k+p$ becomes a Lie algebra. Let us give some illustrations of this situation:

(A) $r=s=0$. In this case $p$ is a supplementary abelian ideal, and $k$ acts as linear transformations on $p$. In other words, $g$ is the semidirect product of $k$ and $p$ where $k$ is a Lie algebra with a given linear representation of $k$ on $p$. Any such linear representation of $k$ gives rise to $g$ which is called the associated affine algebra.

(B) $r=0$. Here all that is assumed is that $p$ is a supplementary ideal to $k$. An important illustration of this situation is the case of the Gallilean group. Recall that the Gallilean group can be regarded as the group of all five by five matrices of the form 


$$
\left(\begin{array}{lll}
A & v & x \\
0 & 1 & t \\
0 & 0 & 1
\end{array}\right)
$$

where $A \in O(3), v \in \mathbf{R}^{3}, x \in \mathbf{R}^{3}$ and $t \in \mathbf{R}$. Such a matrix carries the space time point $\left(x_{0}, t_{0}\right)$ into the space time point $\left(A x_{0}+x+t_{0} v, t+t_{0}\right)$. The corresponding Lie algebra consists of all matrices of the form

$$
\left(\begin{array}{lll}
a & v & x \\
0 & 0 & t \\
0 & 0 & 0
\end{array}\right)
$$

where $a \in o(3)$ and $v, x, t$ as before. Here we can take $k \sim o(3)$ to consist of the subalgebra with $x=v=t=0$ and $p$ to be the seven dimensional subalgebra with $a=0$. Denoting an element of $p$ by $(v, x, t)$ we see that

$$
\left[(v, x, t),\left(v^{\prime}, x^{\prime}, t^{\prime}\right)\right]=s\left((v, x, t),\left(v^{\prime}, x^{\prime}, t^{\prime}\right)\right)=\left(0, t^{\prime} v-t v^{\prime}, 0\right)
$$

and $\xi \cdot(v, x, t)=(\xi \cdot v, \xi \cdot x, 0)$ where $\xi \cdot v$ denotes the usual action of $\xi \in o(3)$ on $v \in \mathbf{R}^{3}$ and similarly for $\xi \cdot x$.

(C) The case where $g$ is semisimple and $k, p$ corresponds to a Cartan decomposition. Here $s=0$.

(D) The case where $k$ is an ideal. Here the action of $k$ on $p$ is trivial. For example, in the case of the Heisenberg algebra we can take $k$ to be the center. For this case $p$ is a symplectic vector space, $k=\mathbf{R}$ acts trivially on $p$ and $r$ is the symplectic two form, while $s=0$.

Let $f \in \Lambda^{2} g^{*}$ be a two form. Identifying $\wedge^{2} g^{*}$ with $\wedge^{2} k^{*}+k^{*} \otimes p^{*}+$ $\wedge^{2} p^{*}$ allows us to write $f=a+b+c$ so that

$$
f\left(\xi+\eta, \xi^{\prime}+\eta^{\prime}\right)=a\left(\xi, \xi^{\prime}\right)+b\left(\xi, \eta^{\prime}\right)-b\left(\xi^{\prime}, \eta\right)+c\left(\eta, \eta^{\prime}\right) .
$$

Now $d f \in \Lambda^{3} g^{*}$ is given by $d f\left(\chi, \chi^{\prime}, \chi^{\prime \prime}\right)=\& f\left(\left[\chi, \chi^{\prime}\right], \chi^{\prime \prime}\right)$ where $\&$ denotes cyclic sum. Writing $\chi=\xi+\eta$ etc., the equation $d f=0$ becomes

$$
\begin{aligned}
& \&\left\{a\left(\left[\xi, \xi^{\prime}\right]+r\left(\eta, \eta^{\prime}\right), \xi^{\prime \prime}\right)+b\left(\left[\xi, \xi^{\prime}\right]+r\left(\eta, \eta^{\prime}\right), \eta^{\prime \prime}\right)\right. \\
& \left.\quad-b\left(\xi^{\prime \prime}, \xi \cdot \eta^{\prime}-\xi^{\prime} \cdot \eta+s\left(\eta, \eta^{\prime}\right)\right)+c\left(\xi \cdot \eta-\xi^{\prime} \cdot \eta+s\left(\eta, \eta^{\prime}\right), \eta^{\prime \prime}\right)\right\}=0 .
\end{aligned}
$$

We now derive various identities for $a, b$, and $c$ by considering special cases of this identity.

$$
\xi=\xi^{\prime}=\xi^{\prime \prime}=0 . \text { In this case the identity becomes }
$$


(*)

$$
\&\left\{b\left(r\left(\eta, \eta^{\prime}\right), \eta^{\prime \prime}\right)+c\left(s\left(\eta, \eta^{\prime}\right), \eta^{\prime \prime}\right)\right\}=0 .
$$

For the case of the affine algebra this identity is vacuous. If $p$ is a subalgebra so that $r=0$, only the identity involving $c$ remains. For example, a direct computation in the case of the Gallilean group shows that $(*)$ reduces to the condition $c\left((0, x, 0),\left(0, x^{\prime}, 0\right)\right)=0$. For the case of the Cartan decomposition only the identity involving $b$ remains. Similarly for the case of the Heisenberg algebra.

$\xi=\xi^{\prime}=0, \eta^{\prime \prime}=0$. In this case the identity becomes

$(* *) \quad a\left(r\left(\eta, \eta^{\prime}\right), \xi^{\prime \prime}\right)-b\left(\xi^{\prime \prime}, s\left(\eta, \eta^{\prime}\right)\right)+c\left(\xi^{\prime \prime} \cdot \eta, \eta^{\prime}\right)+c\left(\eta, \xi^{\prime \prime} \cdot \eta^{\prime}\right)=0$.

For the case of the affine algebra both $r$ and $s$ vanish and this identity becomes

$$
c\left(\xi \cdot \eta, \eta^{\prime}\right)+c\left(\eta, \xi \cdot \eta^{\prime}\right)=0
$$

which asserts that the antisymmetric form $c$ is invariant under the action of $k$. For example, in the case of the Poincare algebra where $k=o(3,1)$ and $p=\mathbf{R}^{4}$ there is no invariant antisymmetric form so we conclude that $c=0$.

In the case that we only assume that $p$ is a subalgebra so that $r$ vanishes the identity becomes

$$
c\left(\xi \cdot \eta, \eta^{\prime}\right)+c\left(\eta, \xi \cdot \eta^{\prime}\right)=b\left(\xi, s\left(\eta, \eta^{\prime}\right)\right) .
$$

For example, in the case of the Gallilean algebra if we apply this identity to $\eta=(v, x, 0)$ and $\eta^{\prime}=\left(v^{\prime}, x^{\prime}, 0\right)$ the right-hand side vanishes and we conclude that $c$, when restricted to $\left(\mathbf{R}^{3}+\mathbf{R}^{3}\right) \wedge\left(\mathbf{R}^{3}+\mathbf{R}^{3}\right)$ is invariant under the action of $o(3)$, which acts diagonally on $\mathbf{R}^{3}+\mathbf{R}^{3}$. There is obviously only one such invariant (up to scalar multiples) and it is given by

$$
c\left((v, x, 0),\left(v^{\prime}, x^{\prime}, 0\right)\right)=m\left(\left\langle v, x^{\prime}\right\rangle-\left\langle v^{\prime}, x\right\rangle\right)
$$

where $\langle$,$\rangle denotes the Euclidean scalar product. If we take \eta=(0, x, 0)$ and $\eta^{\prime}=(0,0, t)$ the right side of $(* *)_{\mathrm{B}}$ still vanishes. On the left the term $\xi \cdot \eta^{\prime}$ vanishes and $\xi \cdot x$ is arbitrary. We conclude that $c((0, x, 0),(0,0, t))=0$. Thus

$$
c\left((v, x, t),\left(v^{\prime}, x^{\prime}, t^{\prime}\right)\right)=m\left(\left\langle v, x^{\prime}\right\rangle-\left\langle v^{\prime}, x\right\rangle\right)+\left\langle l, t^{\prime} v-t v^{\prime}\right\rangle
$$

for some $l \in \mathbf{R}^{3}$ where $(* *)_{\mathrm{B}}$ implies that $\langle l, \xi \cdot v\rangle=b(\xi,(0, v, 0))$. In the case of a Cartan decomposition, or, more generally when $s=0$, the identity (**) becomes

$$
c\left(\xi \cdot \eta, \eta^{\prime}\right)+c\left(\eta, \xi \cdot \eta^{\prime}\right)=a\left(\xi, r\left(\eta, \eta^{\prime}\right)\right) .
$$

For the case where $k$ is an ideal $(* *)$ becomes

$$
a\left(\xi, r\left(\eta, \eta^{\prime}\right)\right)+b\left(\xi, s\left(\eta, \eta^{\prime}\right)\right)=0 .
$$


$\xi=0, \eta^{\prime}=\eta^{\prime \prime}=0$. In this case neither $a$ nor $c$ contributes and we obtain the identity

$$
b\left(\left[\xi^{\prime}, \xi^{\prime \prime}\right], \eta\right)+b\left(\xi^{\prime \prime}, \xi^{\prime} \cdot \eta\right)-b\left(\xi^{\prime}, \xi^{\prime \prime} \cdot \eta\right)=0 .
$$

This identity says that the map from $k$ to $p^{*}$ sending $\xi \rightsquigarrow b(\xi, \cdot)$ is a cocycle. If $k$ is semisimple, then Whitehead's lemma asserts that $b$ must be a coboundary, i.e. that there exists a $\theta \in p^{*}$ such that

$$
b(\xi, \eta)=\theta(\xi \cdot \eta)
$$

Suppose that instead of assuming that $k$ is semisimple we assume that $k$ contains an element in its center which acts as the identity transformation on $p$. Taking $\xi^{\prime}$ to be this element and $\xi^{\prime \prime}$ to be an arbitrary $\xi$ in $(* * *)$ we see that $(* * *)_{\mathrm{S}}$ holds with $\theta(\eta)=b\left(\xi^{\prime}, \eta\right)$. Thus

if either $k$ is semisimple or $k$ contains an element in its center acting as the identity transformation on $p$ then $(* * *)_{\mathrm{S}}$ holds.

For example, in the case of the Gallilean algebra, we see that the bilinear form $b$ is given by

$$
b(\xi,(v, x, t))=\left\langle l^{\prime}, \xi \cdot v\right\rangle+\langle l, \xi \cdot x\rangle
$$

where $l^{\prime}$ and $l$ are elements of $\mathbf{R}^{3}$.

$\eta=\eta^{\prime}=\eta^{\prime \prime}=0$. In this case we simply obtain the identity which asserts that $a$ is a cocycle in $\Lambda^{2} k^{*}$. Again, if $k$ is semisimple we can conclude that $a$ must be a coboundary. In the case of the Gallilean algebra we have thus established that the most general cocycle can be written as

$$
\begin{aligned}
f\left((\xi, v, x, t),\left(\xi^{\prime}, v^{\prime}, x^{\prime}, t^{\prime}\right)\right)= & \tau\left(\left[\xi, \xi^{\prime}\right]\right)+\left\langle l^{\prime}, \xi v^{\prime}-\xi^{\prime} v\right\rangle \\
& +\left\langle l, \xi x^{\prime}-\xi^{\prime} x+t^{\prime} v-t v^{\prime}\right\rangle+m\left(\left\langle v, x^{\prime}\right\rangle-\left\langle v^{\prime}, x\right\rangle\right)
\end{aligned}
$$

where $\tau \in o(3)^{*}$. Now the sum of the first three terms can be written as $\theta\left(\left[(\xi, v, x, t),\left(\xi^{\prime}, v^{\prime}, x^{\prime}, t^{\prime}\right)\right]\right)$ where $\theta=\left(\tau, l^{\prime}, l, 0\right) \in g^{*}$, i.e. as a coboundary. On the other hand, it is clear that the last term is definitely not a coboundary. We have thus recovered the result of Bargmann [1].

If $G$ is the Gallilean group that $H^{2}(g)$ is one dimensional and, up to coboundaries, a cocycle can be written as $f\left((\xi, v, x, t),\left(\xi^{\prime}, v^{\prime}, x^{\prime}, t^{\prime}\right)\right)=m\left(\left\langle v, x^{\prime}\right\rangle-\left\langle v^{\prime}, x\right\rangle\right)$.

We now turn to the problem of describing the action of $G$ on the space of two-cycles in order to determine when two such cycles define equivalent symplectic structures. We begin with the case of the semidirect product, i.e. the affine 
algebra. Every element of the simply connected group corresponding to $g$ can be written as $m \exp \eta$ where $m \in K=\exp k$ and $\eta \in p$. Now $K$ leaves both $k$ and $p$ invariant so that the action of $K$ on $f=a+b+c$ does not mix the summands and the action on each summand is the appropriate exterior or tensor product of the contragredient representation. In case $f=d \theta$ for $\theta \in g^{*}=k^{*}+p^{*}, m f=$ $d m \theta$ where $K$ acts on $g^{*}$ via the contragredient representation. We must therefore examine the action of $\exp \eta$. Now

$$
\left[\eta, \eta^{\prime}\right]=0 \text { and }[\eta, \xi]=-\xi \cdot \eta \text {. }
$$

Thus

$$
\operatorname{Ad}_{\exp -\eta}\left(\xi^{\prime}+\eta^{\prime}\right)=\exp (\operatorname{ad}-\eta)\left(\xi^{\prime}+\eta^{\prime}\right)=\left(\xi^{\prime}+\xi^{\prime} \cdot \eta+\eta^{\prime}\right) .
$$

Therefore

$$
\begin{aligned}
& (\operatorname{Exp} \eta) f\left(\xi^{\prime}+\eta^{\prime}, \xi^{\prime \prime}+\eta^{\prime \prime}\right)=f\left(\operatorname{Exp}-\eta\left(\xi^{\prime}+\eta^{\prime}\right), \operatorname{Exp}-\eta\left(\xi^{\prime \prime}+\eta^{\prime \prime}\right)\right) \\
& \quad=f\left(\xi^{\prime}+\xi^{\prime} \cdot \eta+\eta^{\prime}, \xi^{\prime \prime}+\xi^{\prime \prime} \cdot \eta+\eta^{\prime \prime}\right) \\
& \quad=a\left(\xi^{\prime}, \xi^{\prime \prime}\right)+b\left(\xi^{\prime}, \xi^{\prime \prime} \cdot \eta\right)-b\left(\xi^{\prime \prime}, \xi^{\prime} \cdot \eta\right)+c\left(\xi^{\prime} \cdot \eta, \xi^{\prime \prime} \cdot \eta\right)+c\left(\eta^{\prime}, \eta^{\prime \prime}\right) .
\end{aligned}
$$

Now by $(* * *)$

$$
b\left(\xi^{\prime}, \xi^{\prime \prime} \cdot \eta\right)-b\left(\xi^{\prime \prime}, \xi^{\prime} \cdot \eta\right)=b\left(\left[\xi^{\prime}, \xi^{\prime \prime}\right], \eta\right)
$$

and, by $(* *)_{\mathrm{A}}$

$$
c\left(\xi^{\prime} \cdot \eta, \xi^{\prime \prime} \cdot \eta\right)=-c\left(\eta, \xi^{\prime} \cdot \xi^{\prime \prime} \cdot \eta\right)=c\left(\xi^{\prime} \cdot \xi^{\prime \prime} \cdot \eta, \eta\right)=1 / 2 c\left(\left[\xi^{\prime}, \xi^{\prime \prime}\right] \cdot \eta, \eta\right) .
$$

We can thus write

$$
(\operatorname{Exp} \eta)(a+b+c)=\left(a+d\left(b_{\eta}+1 / 2 c_{\eta \eta}\right)\right)+\left(b+d c_{\eta}\right)+c
$$

where $b_{\eta}$ and $c_{\eta \eta} \in k^{*}$ are defined by

$$
b_{\eta}(\xi)=b(\xi, \eta) \text { and } c_{\eta \eta}(\xi)=c(\xi \cdot \eta, \eta)
$$

while $c_{\eta} \in p^{*}$ is defined by

$$
c_{\eta}\left(\eta^{\prime}\right)=-c\left(\eta, \eta^{\prime}\right)
$$

In the important special case where $(a+b)=d(\tau+\theta)$ is exact, where $\tau \in k^{*}$ and $\theta \in p^{*}$ we can write

$$
(\operatorname{Exp} \eta)(d(\tau+\theta)+c)=d\left(\left(\tau+b_{\eta}+1 / 2 c_{\eta \eta}\right)+\left(\theta+c_{\eta}\right)\right)+c .
$$

We can therefore describe the situation as follows. The element $c$ is invariant under the action of $G$. It is invariant under Exp $p$ by the above computation and it is invariant under the action of $K$ by $(* *)_{\mathrm{A}}$. For a given choice of $c$ we can move $\theta$ into $\left(h^{*-1}\right) \theta+c_{\eta} h \in K$ and $\eta \in p$. This determines an action of 
$K \times p$ on $p^{*}$. Suppose that we have parametrized the orbits of this action and have, in fact, chosen a cross-section for these orbits. For a given orbit we have thus picked a fixed $\theta$. This determines a subgroup of $G$, the isotropy group of $\theta$. The corresponding algebra consists of those $(\xi, \eta)$ for which $\xi \theta+c_{\eta}=0$. The set of $\xi^{\prime}$ which occur forms a subalgebra of $k$ which we denote by $k_{\theta}$. Thus $\xi \in$ $k_{\theta}$ if and only if there exists an $\eta_{\xi} \in p$ such that $\theta(\xi \cdot \eta)=c\left(\eta_{\xi}, \eta\right)$ for all $\eta \in p$. It is easy to check that the identity $(* *)_{A}$ implies that the assignment $\xi \rightsquigarrow \rightarrow \eta_{\xi}$ is a cocycle of $k_{\theta}$ with values in $p$. If this cocycle is a coboundary (for instance if $k_{\theta}$ is semisimple or contains the identity operator) then we can find an $\bar{\eta}$ such that $\xi \theta-c_{\xi \bar{\eta}}=\xi\left(\theta-c_{\bar{\eta}}\right)=0$. Thus by changing our choice of $\theta$ within the orbit we can arrange that $k_{\theta}$ consist exactly of those $\xi$ for which $\xi \theta=0$. Notice that this equation is equivalent to the equation $\theta_{\eta}(\xi)=0$ for all $\eta$. If we consider the action of $\operatorname{Exp} \eta$ on the $k^{*}$ component, it adds exactly $\theta_{\eta}+1 / 2 c_{\eta \eta}$. If $c_{\eta \eta}=$ 0 , we see that the orbit of $\tau$ is just the complete inverse image of the orbit of $\rho_{\theta}(\tau)$ under $K_{\theta}$ where $\rho_{\theta}: k^{*} \rightarrow k_{\theta}^{*}$ is the projection dual to the injection of $k_{\theta} \rightarrow k$. In this case the cocycles are thus parametrized by $c, \theta$ ranging over a cross-section of the action of $G$ on $k^{*}$ determined by $c$, and $\chi$ ranging over a cross-section for the action of $K_{\theta}$ on $k_{\theta}^{*}$.

For example, for the case of the Lie algebra of the Poincare group we have already seen that $c=0$. The orbits of $G$ on $p^{*}$ are thus the same as the orbits of $K=S O(3,1)$ on $p^{*}$ and consist of single sheeted hyperboloids $\theta^{2}=m^{2}>0$, $\theta_{0}>0$ and $\theta^{2}=m^{2}>0, \theta_{0}<0$; the forward light cone $\theta^{2}=0, \theta_{0}>0$, the backward light cone $\theta^{2}=0, \theta_{0}<0$, the single sheeted hyperboloids $\theta^{2}=-m^{2}$ $<0$; and the origin. We thus choose cross-sections for these orbits as follows:

$$
\begin{array}{ll}
(m, 0,0,0), \quad(-m, 0,0,0), & (1,1,0,0), \\
(-1,1,0,0), \quad(0, m, 0,0), & (0,0,0,0) .
\end{array}
$$

It is easy to see that the group $K_{(m, 0,0,0)}$ is exactly $S O(3)$. Its orbits acting on the dual of its Lie algebra are spheres. If we call $s$ the radius of these spheres, we see that a fanily of orbits is parametrized by the two real parameters $m>0$ and $s \geqslant 0$. Here $m$ is the "mass" and $s$ is the "spin". For "mass zero" i.e. for $(1,1,0,0)$ or $(-1,1,0,0)$ it is easy to see that the corresponding isotropy group is the Euclidean group $E(2)$. The orbits of $E(2)$ in the dual of its Lie algebra are easily seen to be cylinders (of radius $r$, say) and points on the axis $r=0$. If we let the real parameter $s$ describe the points on this axis we see that the symplectic structures corresponding to $(1,1,0,0)$ are parametrized by $r>0$ and, if $r=0$ by an arbitrary real parameter $s$. The case $r>0$ does not arise in known physical systems; for $r=0$ the parameter $s$ is also called the "spin". The isotropy group of 
$(0, m, 0,0)$ is $S l(2)$. Its orbits are again hyperboloids, forward and backward "light cones" and the origin. No particles with negative mass ${ }^{2}$ ("tachyons") seem to occur.

Let us now do a slightly more complicated computation determining the symplectic homogeneous spaces for the Gallilean group. Here $p$ is not abelian. However, it is easy to check that

$$
\operatorname{Ad}(\exp -(0, v, x, t))(\xi, w, y, s)=(\xi, w+\xi v, y+\xi(x+1 / 2 t v)+t w-s v, s) \text {. }
$$

We have already written the form of the most general cocycle of the Gallilean algebra. Under the action of $\exp (\eta)$ it is easy to check that $l^{\prime}$ is moved into $l^{\prime}+$ $m x$ and $l$ is changed into $l-m v$. Thus by suitable choice of $x$ and $v$ we can arrange that both $l$ and $l^{\prime}$ vanish, provided that $m \neq 0$. (In the case that $m \neq 0$ one can show that the physical interpretation of $l$ is the linear momentum of the "center of mass". Our choice of $v$ amounts to making a change to a new frame of reference in which the center of gravity is at rest. The physical interpretation of $-l^{\prime} / m$ is that it is the position of the center of mass in the frame in which it is at rest. By shifting the origin of the coordinate system we can arrange that this is the origin.) Once we have arranged that $l=l^{\prime}=0$, the only possibility left for $\eta$ (in the case of nonzero $m$ ) is $\eta=(0,0, t)$ and it is clear that $\exp (0,0,-t)$ acts trivially. Thus we are left with the action of $S O(3)$ on $k^{*}=$ $o(3)^{*}$. Again, the orbits are spheres, parametrized by their radius, a nonnegative parameter, $s$. Thus for $m \neq 0$ the homogeneous symplectic manifolds for the Gallilean group are parametrized by $m$ and $s \geqslant 0$, the "mass" and the spin. For $m=$ 0 , we cannot change while $l^{\prime}$ is moved into $l^{\prime}+t l$. On the other hand, $\tau$ is moved into $\tau+\left\langle l^{\prime}, \cdot v\right\rangle+\langle l \cdot \cdot(x+1 / 2 t v)\rangle$. If we identify $\tau$ as a vector in three space this last expression can be written as $\tau+l^{\prime} \times v+l \times(x+1 / 2 t v)$ where $\times$ denotes vector product. In this case it is more convenient to let $G$ act by letting $S O(3)$ act first and then $\exp p$. By applying a suitable element of $S O(3)$ we can arrange that $l=(f, 0,0)$ and then, if $f \neq 0$ that $l^{\prime}=(0, b, c)$. If $l$ and $l^{\prime}$ are independent by suitable choice of $v$ and $x$ we can arrange that $\tau=0$. If $f \neq 0$ and $b=c=0$, then we can arrange that $\tau=( \pm s f, 0,0)$ where $s \geqslant$ and $f>0$. (This corresponds to the case of a particle of zero mass, travelling with infinite velocity. Here the condition that $b=c=0$ amounts to the condition that the "disturbance is transverse" and the parameter $f$ is the "inverse of the wave length", i.e. the "color". The parameter $s$ is called the spin and the for - is called the helicity. For details, see Souriau [5, p. 195].)

We can compute the space of cocycles, and the corresponding symplectic manifolds for the Gallilean group from a slightly different point of view. Let $S O(3) \times \mathbf{R}^{3}$ act on $\mathbf{R}^{4}$ by $(A, v) \cdot(x, t)=(A x+t v, t)$. Here $(x, t)$ is a vector in $\mathbf{R}^{4}$, 
with $x$ a vector in $\mathbf{R}^{3}$ and $t$ in $\mathbf{R}$. We can regard the Gallilean group as the semidirect product of $S O(3) \times \mathbf{R}^{3}$ with $\mathbf{R}^{4}$. Again we have a $(k, p)$ decomposition but this time with $k=o(3)+\mathbf{R}^{3}$ (semidirect) and $p=\mathbf{R}^{4}$. It is easy to check that there are no invariant antisymmetric two forms on $\mathbf{R}^{4}$, so that $c=0$. We can write $b$ as

$$
b=b_{1}(\xi, x)+b_{2}(\xi, t)+b_{3}(v, x)+b_{4}(v, t)
$$

where $\xi$ is in $o(3)$ and $v$ in $\mathbf{R}^{3}$. Condition (***) implies that

$$
b_{3}(\xi \cdot v, x)+b_{4}(\xi \cdot v, t)=b_{1}(\xi, t)-b_{3}(v, \xi \cdot x)
$$

and

$$
b_{1}\left(\left[\xi, \xi^{\prime}\right], x\right)+b_{2}\left(\left[\xi, \xi^{\prime}\right], t\right)=b_{1}\left(\xi, \xi^{\prime} \cdot x\right)-b_{1}\left(\xi^{\prime}, \xi \cdot x\right) .
$$

The first of these equations, with $t=0$, implies that $b_{3}(v, x)=m\langle v, x\rangle$ while for $t \neq 0$ it implies that $\left.b_{4}(\xi \cdot v, t)=b_{1}(\xi, t)\right)$. The second equation implies that $b_{1}$ is a cocycle of $o(3)$ with values in $\mathbf{R}^{3^{*}}$, and hence a coboundary, and that $b_{2}=0$. Thus

$$
b=\langle l, \xi \cdot x+t v\rangle+m\langle v, x\rangle .
$$

The fact that $a$ is a cocycle in $\Lambda^{2} k^{*}$ implies that it is a coboundary, $a=d\left(\tau+l^{\prime}\right)$ where $\tau \in o(3)^{*}$ and $l \in \mathbf{R}^{3 *}$. We thus recover the expression that we derived above for the most general cocycle for the Gallilean algebra. The analysis of the operation of the Gallilean group on the space of cocycles proceeds as before.

Let us now do a computation at the opposite extreme-the Heisenberg algebra. In this case $k$ is the one dimensional center that we may identify with $\mathbf{R}$, and $p$ is a symplectic vector space with $r$ identified as its symplectic two form. The action of $k$ on $p$ is trivial and $s=0$. Since $k$ is one dimensional, $a=0$ and conditions $(* *)$ and $(* * *)$ are vacuous. Condition $(*)$ can be interpreted as follows: let $\omega$ denote the symplectic form on $p$ and let $\kappa \in p^{*}$ be defined by $\kappa=b(1, \cdot)$. Then $(*)$ becomes $\omega \wedge \kappa=0$. If $\operatorname{dim} p=2$, this imposes no condition. If $\operatorname{dim} p>2$, then this implies $\kappa=0$. Indeed, for $\operatorname{dim} p>2$ we can write $\omega=\theta \wedge \kappa+\omega^{\prime}$ for some suitable $\theta \in p^{*}$ and where $\omega^{\prime} \wedge \kappa \neq 0$. Any $c \in \wedge^{2} p^{*}$ gives a cocycle. The element $\omega \in \Lambda^{2} p^{*}$ is a coboundary, $\omega=d l$ where $l$ is any element of $g^{*}$ satisfying $l(1)=1$, with 1 denoting the basis element of the center which we have identified with $\mathbf{R}$. It is clear that the only coboundaries are the multiples of $\omega$. Now

$$
[(s, v),(t, w)]=(\omega(v, w), 0) \quad \text { where } s, t \in k=\mathbf{R} \text { and } v, w \in p .
$$

Therefore

$$
\operatorname{Ad}(\exp (s, v))(t, w)=(t+\omega(v, w), w)
$$


Thus the group acts trivially on $\bigwedge^{2} p^{*}$. For $\operatorname{dim} p=2$ it maps a nonzero $b \in k^{*}$ $\otimes p^{*}$ onto $b+c$ where $c$ ranges all over $\Lambda^{2} p^{*}$. For $\operatorname{dim} p>2$ the action of the group on the space of cocycles is completely trivial. Thus $H^{2}(g) \sim k^{*} \otimes p^{*}$ for $\operatorname{dim} p=2$ while $H^{2}(g) \sim \wedge^{2} p^{*} /\{\omega\}$ for $\operatorname{dim} p>2$. The orbits of $G$ acting on $g^{*}$ via the contragredient representation are the hyperplanes $l(1,0)=$ const. for $l(1,0) \neq 0$, and the points in the subspace $l(1,0)=0$. Thus these orbits either have dimension equal to $\operatorname{dim} p$ or are zero dimensional. The symplectic manifolds corresponding to the nonvanishing cohomology classes for $\operatorname{dim} p=2$ are all two dimensional while for $\operatorname{dim} p>2$ the dimension corresponding to an element of $\bigwedge^{2} p^{*}$ is equal to its rank.

3. We now list the homogeneous symplectic manifolds for the low dimensional Lie algebras.

$n=1$. There is only one Lie algebra of dimension one, the trivial Lie algebra, the corresponding simply connected group is just the additive group of real numbers, which obviously does not act transitively on any symplectic manifold of positive dimension. Hence the only homogeneous symplectic manifold is a point. Nevertheless, even in this most trivial example there are a number of interesting lessons to be learned. The action of the adjoint group is trivial, and hence so is the coadjoint action. Thus the orbits of $G$ in $g^{*}$ consist entirely of points. This is obviously the case for any commutative Lie algebra. Although the orbits are all distinct, they all correspond to the same symplectic manifold, because the operator $d$ is trivial, and hence all orbits give the zero cocycle. From the point of view of "classical mechanics" these orbits are all the same. But from the "quantum" viewpoint, i.e. from the point of view of representation theory, they all correspond to different infinitesimal characters, to different representations. This is already true at the level of "prequantization", to use Kostant's [4] terminology, where one is interested in classifying homogeneous Hermitian line bundles with connection. Another comment is in order, even at the level of "classical mechanics". While it is obvious that the real line cannot act transitively on any manifold whose dimension is greater than one, the action can be "ergodic" in any of the various senses, e.g. topologically transitive, or metrically transitive, or mixing, etc. Each of these concepts corresponds to a different mathematical formulation of the intuitive notion of "irreducibility" for a mechanical system. The notion of transitivity, the one we are dealing with, is just the most simple of these concepts.

$n=2$. There are two Lie algebras of dimension two, the trivial Lie algebra and the Lie algebra with basis $\{\xi, \eta\}$ and bracket relations $[\xi, \eta]=\eta$. We shall call this second algebra the scale algebra. It corresponds to the group of symmetries 
in which one can change the origin of measurement (time translation) and the choice of units (scaling). For both algebras all two forms are cocycles since the algebra is two dimensional. However the operator $d: g^{*} \rightarrow \bigwedge^{2} g^{*}$ behaves differently in each of the two cases.

The trivial algebra. Here the operator $d$ is trivial. Thus each element of $\wedge^{2} g^{*}$ represents a different cohomology class and the action of $G$ on $\wedge^{2} g^{*}$ is trivial. Each element of $\wedge^{2} g^{*}$ gives a distinct symplectic space. It is easy to see that the explicit realizations of these spaces are given by $\omega=c d x \wedge d y, c \neq 0$, with $\xi \rightarrow \partial / \partial x$ and $\eta \rightarrow \partial / \partial y$. In addition, of course, there is the zero dimensional symplectic manifold corresponding to the orbits of $g^{*}$ to which the remarks made above in the one dimensional case apply.

The scale algebra. We have a $k+p$ decomposition with both $k$ and $p$ one dimensional. Hence $a=c=0$, and, since $d$ is nontrivial (or by applying the general argument using the fact that $k$ contains the identity), we know that every cocycle is a coboundary. Thus every $b$ has the form $b(\xi, \cdot)=l([\xi, \cdot])$ for some $l \in p^{*}$. The group $K$ acts on $p$ and hence on $p^{*}$ by multiplication by positive numbers, while exp $p$ does not change $d l$. Hence there are three symplectic homogeneous spaces corresponding to the alternatives $l(\eta)>0, l(\eta)=0$, and $l(\eta)<0$. Let $(a, b)$ be the coordinates on $g^{*}$ given by $\xi$ and $\eta$, so that $\theta(\xi)=a$ and $\theta(\eta)=$ $b$ if $\theta \in g^{*}$ has coordinates $(a, b)$. Then a direct computation shows that

$$
\operatorname{Ad}_{\exp t \xi}^{\#}(a, b)=\left(a, e^{-t} b\right) \text { and } \operatorname{Ad}_{\exp t \eta}^{\#}(a, b)=(a+t b, b) \text {. }
$$

For $b>0$, say, we get a symplectic manifold, and $\hat{\xi}=b(\partial / \partial b), \hat{\eta}=-b(\partial / \partial a)$. It is clear that the invariant two form $\omega$ must be given by $\omega=\kappa b^{-1} d b d a$ for $\kappa \neq 0$. On the other hand, replacing $(a, b)$ by $(s a, s b)$, where $s$ is an arbitrary constant, does not change $\hat{\xi}$ or $\hat{\eta}$, but replaces $\kappa$ by $s \kappa$. This shows that the symplectic manifolds corresponding to $l(\eta)>0$ and $l(\eta)<0$ are equivalent, so that there is exactly one two dimensional homogeneous symplectic manifold for the scale algebra. There is, of course, also the zero dimensional manifold as well.

If we introduce the variables $x=a / b, y=b$, we can rewrite the two dimensional action as

$$
(\exp t \xi)(x, y)=\left(e^{t} x, e^{-t} y\right), \quad(\exp t \eta)(x, y)=(x+t, y) .
$$

In terms of these coordinates we have $\omega=d x d y$.

If we set $u=a$ and $v=-\log b$ we can describe the action as $\omega=d u d v$, $\exp t \xi(u, v)=(u, v+t)$ and $\exp t \eta(u, v)=\left(u+t e^{-v}, v\right)$.

$n=3$. The three dimensional Lie algebras over $R$ are classified as follows (cf. Jacobson, Lie algebras, pp. 11-13):

(i) the trivial algebra, 
(ii) the Heisenberg algebra,

(iii) the direct sum $\mathbf{R}+h$ where $h$ is the two dimensional scale algebra,

(iv) A $_{\mathrm{A}}$ the affine algebra $k+p$ where $k$ is one dimensional and $p$ is two dimensional.

Here, a basis element of $k$ acts on $p$ via the linear transformation $A$, which is nonsingular. Here $A$ is determined only up to conjugacy on $p$ and multiplication by any nonzero real number (since the basis of $k$ was chosen arbitrarily). We will distinguish several possibilities, according to whether the trace of $A$ is or is not zero. If $\operatorname{tr} A=0$, and if $A$ has real eigenvalues then we may arrange that $A$ is diagonal with eigenvalues \pm 1 . Thus $A$ is the matrix which infinitesimally preserves the indefinite metric $x y$ on the $(x, y)$ plane, and $g$ is the algebra of (infinitesimal) motions for this metric. We list this algebra as

(iv) $e(1,1)$.

If $\operatorname{tr} A=0$ and $A$ has complex eigenvalues, then the eigenvalues must be purely imaginary, and we can arrange that they are \pm 1 . Thus $A$ is an infinitesimal rotation for the Euclidean metric in the plane and we are in the case

(v) $e(2)$, the Lie algebra of the group of Euclidean motions in the plane,

(vi) the case of the affine algebra (iv) ${ }_{\mathrm{A}}$ where $\operatorname{tr} A \neq 0$,

(vii) the orthogonal algebra $o(3)$, and

(viii) $\operatorname{sl}(2, \mathbf{R})$.

For the case of the trivial algebra, every element of $\bigwedge^{2} g^{*}$ is a cocycle and no element is a coboundary; each nonzero element has a one dimensional kernel which will act trivially on the corresponding symplectic manifold, which is a homogeneous symplectic manifold for the quotient two dimensional trivial algebra. We have already discussed the Heisenberg algebra. For the case (iii) we can apply the $k, p$ decomposition with $k=\mathbf{R}$ and with $p=h$, both ideals. Then $a=0$ and $c$ is a cocycle for the scale algebra, while condition $(* *)_{D}$ implies that $b(k,[h, h])=0$. The space of possible $b$ 's is thus one dimensional and none of them is a coboundary, and thus $H^{2}(g)$ is one dimensional. Let $x, y$, and $z$ be a basis of $g$ with the bracket relations $[x, y]=y,[x, z]=[y, z]=0$, and let $A_{t}=\exp t x, B_{t}=\exp t y$, and $C_{t}=\exp t z$ be the corresponding one parameter subgroups. Then it follows from the above computation that the space of all two dimensional symplectic manifolds is parametrized by the constant $m=$ $b(z, x)$. The actual manifolds are all $\mathbf{R}^{2}$ with coordinates $(u, v)$ and $\omega=d u \wedge d v$ and actions given by

$$
A_{t}(u, v)=(u, v+t), \quad B_{t}(u, v)=\left(u+t e^{v}, v\right), \quad C_{t}(u, v)=(u+m t, v) .
$$

We now turn to the remaining cases:

(iv) the algebra $e(1,1)$. Here we can choose a basis with $[z, x]=x$, 
$[z, y]=-y$ and $[x, y]=0$. Here $k$ is spanned by $z$ and $p$ is spanned by $x$ and y. Any $c$ in $\Lambda^{2} p^{*}$ is a cocycle and is not a coboundary if $c \neq 0$. The operator $d: g^{*} \rightarrow \Lambda^{2} g^{*}$ has a one dimensional kernel and hence the space of coboundaries is two dimensional. Thus $d g^{*}=k^{*} \otimes p^{*}$, i.e. all $b$ 's are coboundaries. If $c=0$, the map of $p \rightarrow k^{*} \otimes p^{*}$ sending $u m \rightarrow c(\cdot v, \cdot)$ is nonsingular and hence surjective. Thus if $c \neq 0$, we can eliminate $b$ by the action of $G$. There are thus two families of two dimensional symplectic manifolds, one parametrized by nonzero $c \in \Lambda^{2} p^{*}$ and the other parametrized by a cross-section for the orbits of $C_{t}$ acting on $p^{*}$. The first family all consist of $\mathbf{R}^{2}$ with coordinates $(u, v)$ and with varying forms $\omega=m d u \wedge d v$ (where $m=c(x, y)$ ) and action

$$
A_{t}(u, v)=(u+t, v), \quad B_{t}(u, v)=(u, v+t), \quad C_{t}(u, v)=\left(e^{t} u, e^{-t} v\right) .
$$

To describe the orbits in $p^{*}$ observe that $x$ and $y$ can be thought of as functions on $g^{*}$ and hence on $p^{*}$, and the orbits of $C_{t}$ are the various components of the hyperbolas $x y=\kappa$ for different values of the constant $\kappa$. The actual orbits of $G$ in $g^{*}$ are given by the same equations and are, in fact, just cylinders over these curves with generators in the $k^{*}$ direction. Again the orbits can all be identified with $\mathbf{R}^{2}$, with coordinates $(u, v)$ and $\omega=d u \wedge d v$. For the orbits on which $x \neq$ 0 we can use the vectors $(1, \kappa)$ as cross-sections to the orbits; the corresponding actions are given by

$$
A_{t}(u, v)=\left(u+t e^{-v}, v\right), \quad B_{t}(u, v)=\left(u-\kappa e^{+v}, v\right), \quad C_{t}(u, v)=(u, v+t) .
$$

The case $\kappa=\infty$ is obtained in the limit as $A_{t}$ acting as the identity and $B_{t}(u, v)$ $=\left(u \pm t e^{v}, v\right)$ and $C_{t}(u, v)=(u, v+t)$. It is interesting to give some interpretation to the parameters $m$ and $\kappa$. Notice that the algebra $e(1,1)$ contains two copies of the scale algebra, namely $z, x$ and $z, y$, with the group $C_{t}$ multiplying $x$ by $e^{t}$ and multiplying $y$ by $e^{-t}$. Now there are two situations where making a change in scale of one variable induces the inverse change of scale of a second variable, if the variables are dual to one another (i.e. represent coordinates in dual one dimensional vector spaces) or if the variables are inverse to one another. The first family of orbits corresponds to the duality situation, with the parameter $m$ giving the duality between $u$ and $b$. The second family of orbits corresponds to the situation where the scale algebra is acting on variables $r$ and $s$ related by $r s=\kappa$.

(v) The situation for the Euclidean algebra $e(2)$ is quite similar to that for $e(1,1)$. The cohomology is one dimensional, each nonzero element of $\Lambda^{2} p^{*}$ corresponding to a nonzero cohomology class and giving rise to a symplectic manifold with $x$ and $y$ acting as constant vector fields. The remaining symplectic 
manifolds are given by orbits in $g^{*}$ which are the cylinders $x^{2}+y^{2}=r^{2}$, for $r$ positive, together with the zero dimensional orbits on the $z$ axis.

(vi) For the affine algebra with $\operatorname{tr} A \neq 0$, there is no nonzero invariant in $\Lambda^{2} p^{*}$, and thus the space of cocycles is two dimensional, the cohomology vanishes. The orbits in $g^{*}$ are seen to be cylinders over the orbits of exp $t z$ acting on $p^{*}$, and these provide all the two dimensional symplectic manifolds. The $z$-axis again splits into zero dimensional orbits.

(vii) The orthogonal algebra is semisimple, so its cohomology vanishes. The orbits in $g^{*}$ are given by the spheres $x^{2}+y^{2}+z^{2}=r^{2}$, where $x, y$, and $z$ are the usual basis of $o(3)$, with the bracket relations $[x, y]=z,[z, x]=y,[z, y]$ $=-x$.

(viii) The algebra $\operatorname{sl}(2)$ is also semisimple, so its cohomology also vanishes. We may choose a basis $x, y$, and $z$ with the bracket relations $[x, y]=z,[z, x]$ $=x$ and $[z, y]=-y$. Then $x y+z^{2} / 2$ is invariant under the action of $g$, and thus, when considered as a function on $g^{*}$, defines two dimensional surfaces which are invariant under $G$. The connected components of these level surfaces are clearly the orbits of $G$; they are the single sheeted hyperboloids, the double sheeted hyperboloids, and the two components of the punctured light cone.

We now study the behavior of homogeneous symplectic manifolds under deformation of the Lie algebra structure. As an illustration of what can happen let us consider the deformation of $\operatorname{sl}(2)$ into $e(1,1)$. We consider a three dimensional vector space with basis $x, y$, and $z$, and with bracket relations $[z, x]=x$, $[z, y]=-y,[x, y]=\epsilon z$. For $\epsilon \neq 0$, this algebra is isomorphic to $\operatorname{sl}(2)$, while for $\epsilon=0$ the algebra is $e(1,1)$. For all values of $\epsilon$ the function $x y+\epsilon z^{2} / 2$ is invariant. The double sheeted hyperboloids, corresponding to positive values of this function for $\epsilon<0$ (and to negative values for $\epsilon>0$ ), clearly deform into the cylinders $x y=c$ for $\epsilon=0$. It is interesting to examine the behavior of the single sheeted hyperboloids. They provide both the other cylinders and also the symplectic manifolds of $e(1,1)$ corresponding to nonvanishing cohomology classes of $e(1,1)$. (Recall that $H^{2}(e(1,1))=\mathrm{R}$ while $H^{2}(s l(2))=0$.) Indeed, for a fixed value of $x y+\epsilon z^{2} / 2$ the points near $x=0$ of the hyperboloid (or near $y=0$ ) clearly move off to infinity at the rate $\epsilon^{-1 / 2}$ and the hyperboloid splits into two cylinders. As to the orbits with nonvanishing cohomology, observe that the cocycles are of the form $h x^{*} \wedge y^{*}$, and, for all $\epsilon$, we have $d z^{*}=\epsilon x^{*} \wedge y^{*}$. For $\epsilon=0$ we know that $h x^{*} \wedge y^{*}$ is not a coboundary (for nonvanishing $h$ ) while for $\epsilon \neq 0$ the above equation shows that $h x^{*} y^{*}=d\left(h \epsilon^{-1} z^{*}\right)$. This suggests looking at the orbit through the point with $x=0, y=0$ and $z=h \epsilon^{-1}$, i.e. the orbit $x y+\epsilon z^{2} / 2=m_{\epsilon}$ where $m_{\epsilon}=(2 \epsilon)^{-1} h^{2}$. A direct computation shows that if we consider a bounded region of $x$ and $y$, the action on this portion of 
the orbit tends to the desired limiting action for $e(1,1)$. This computation is suggestive of a similar computation that can be carried out in higher dimensions. According to Segal's theory of the cosmos, the correct group of the universe is the fifteen dimensional conformal group $S O(4,2)$ (or one of its covering groups). If we let $\epsilon=R^{-1}$ where $R$ is the radius of the cosmos in laboratory units, then as $R \rightarrow \infty$ the Lie algebra $o(4,2)$ deforms into a fifteen dimensional algebra consisting of the semidirect product of the Lorentz algebra plus scale acting on $V+V^{*}$ where $V$ is the four dimensional Minkowski space. The cohomology of this limiting algebra is again one dimensional, the classes being parametrized by cocycles of the form $c=h\left(\left\langle v, v^{\prime}\right\rangle-\left\langle v^{\prime}, v^{*}\right\rangle\right)$ where $\left(v, v^{*}\right)$ and $\left(v^{\prime}, v^{*}\right)$ lie in $V+V^{*}$. The maximal orbits are twelve dimensional, and for the orbits with nonvanishing cohomology the parameter $h$ plays the role similar to Planck's constant. The maximal orbits with zero cohomology are again twelve dimensional, given by three parameters. By picking a suitable representative point on one of these orbits, and letting the subgroup consisting of the ten dimensional Poincare group act, one gets an eight dimensional orbit. The mass parameter of this eight dimensional orbit of the Poincare group can be related to the parameters describing the twelve dimensional orbit of the fifteen dimensional algebra. This suggests a possible relationship between quantization, the masses of the observed particles and cosmology.

\section{BIBLIOGRAPHY}

1. V. Bargmann, On unitary ray representations of continuous groups, Ann. of Math. (2) 59 (1954), 1-46. MR 15, 397. $145-159$.

2. B.-Y. Chu, Symplectic homogeneous spaces, Trans. Amer. Math. Soc. 197 (1974),

3. M. Duflo and $M$. Vergne, Une propriété de la représentation coadjointe d'une algèbre de Lie, C. R. Acad. Sci. Paris Sér. A-B 268 (1969), A583-A585. MR 39 \#6935.

4. B. Kostant, Quantization and unitary representations, Lecture Notes in Math., vol. 170, Springer-Verlag, Berlin and New York, 1970, pp. 87-208.

5. J.-M. Souriau, Structures des systèmes dynamiques, Maîtrises de mathématiques, Dunod, Paris, 1970. MR 41 \#4866.

DEPARTMENT OF MATHEMATICS, HARVARD UNIVERSITY, CAMBRIDGE, MASSACHUSETTS 02138 